\title{
Review
}

\section{Life and death partners: apoptosis, autophagy and the cross-talk between them}

\author{
A Eisenberg-Lerner ${ }^{1}$, S Bialik ${ }^{1}$, H-U Simon ${ }^{2}$ and A Kimchi ${ }^{\star 1}$
}

It is not surprising that the demise of a cell is a complex well-controlled process. Apoptosis, the first genetically programmed death process identified, has been extensively studied and its contribution to the pathogenesis of disease well documented. Yet, apoptosis does not function alone to determine a cell's fate. More recently, autophagy, a process in which de novo-formed membrane-enclosed vesicles engulf and consume cellular components, has been shown to engage in a complex interplay with apoptosis. In some cellular settings, it can serve as a cell survival pathway, suppressing apoptosis, and in others, it can lead to death itself, either in collaboration with apoptosis or as a back-up mechanism when the former is defective. The molecular regulators of both pathways are inter-connected; numerous death stimuli are capable of activating either pathway, and both pathways share several genes that are critical for their respective execution. The cross-talk between apoptosis and autophagy is therefore quite complex, and sometimes contradictory, but surely critical to the overall fate of the cell. Furthermore, the crosstalk is a key factor in the outcome of death-related pathologies such as cancer, its development and treatment.

Cell Death and Differentiation (2009) 16, 966-975; doi:10.1038/cdd.2009.33; published online 27 March 2009

\begin{abstract}
Autophagy, a process long known to provide a survival advantage to cells undergoing nutrient deprivation or other stresses, has also been more recently linked to the actual death process itself. Thus apoptosis is not the sole means by which the cell can undergo a genetically programmed regulated process by which it undergoes self-elimination. Cell death can occur by several mechanisms and the phenotypic changes that accompany cell death can vary depending on the stimulus and cell setting. In any given death scenario, the cell decides which pathway to use, depending on the nature of the stimulus and the particulars of the cell environment. Furthermore, apoptosis and autophagy are not mutually exclusive pathways. They have been shown to act in synergy and also to counter each other. They share many of the same molecular regulators. In a clinical setting, one cannot predict the outcome of inhibition or activation of one death program without considering the effect on the other. This review will focus on the cross-talk between the autophagic and apoptotic pathways, with an analysis of how this may affect the clinical applications of death suppression/activation to cancer. The process of necrosis, the third means by which the cell can undergo a genetically programmed self-elimination, will not be discussed in detail. Although not intended to provide an exhaustive summary of the recent literature, the discussion will include salient experimental results as
\end{abstract}

examples of the different facets of the apoptosis/autophagy interplay.

An issue that has been raised and discussed in the literature is that in many cell settings, autophagy accompanies, rather than causes, cell death. ${ }^{1}$ This argument is based on the fact that many studies that claim autophagic cell death prove that autophagy occurs, and that death ensues, but do not rigorously show that one causes the other. The observation that inhibition of autophagy results in significant attenuation of cell death is stronger evidence for autophagic cell death, as long as this manipulation is specific for autophagy. For example, studies that knock down or delete specific autophagy genes such as Atg7 are more valid than those that target only Atg5, which has also been shown to regulate apoptosis (see below), or those that use drugs such as the $\mathrm{PI}(3) \mathrm{K}$ inhibitor 3-MA or inhibitors of lysosomal function in general. Furthermore, these studies must verify that apoptotic cell death, which often accompanies autophagy, is not affected by these manipulations, by verifying that markers of apoptosis, such as caspase activity, chromatin degradation and so on, are not suppressed by autophagy inhibition. Although many studies fail to achieve these criteria, numerous studies do clearly indicate the presence of bona fide autophagic cell death, and we will assume the position that both apoptosis and autophagy are legitimate means of self-killing.

\footnotetext{
${ }^{1}$ Department of Molecular Genetics, Weizmann Institute of Science, Rehovot 76100, Israel and ${ }^{2}$ Faculty of Medicine, Institute of Pharmacology, University of Bern, $\mathrm{CH}-3010$ Bern, Switzerland

*Corresponding author: A Kimchi, Department of Molecular Genetics, Weizmann Institute of Science, Herzel, Rehovot 76100, Israel. Tel: +972 89342428 ;

Fax: + 9728 9315938; E-mail: adi.kimchi@weizmann.ac.il

Keywords: autophagy; apoptosis; tumorigenesis

Abbreviations: AMPK, AMP-activated kinase; DAPk, death-associated protein kinase; DRAM, damage-regulated modulator of autophagy; ER, endoplasmic reticulum; JNK, c-jun N-terminal kinase; MEFs, mouse embryonic fibroblasts; MLC, myosin II regulatory light chain; MOMP, mitochondrial outer membrane permeability; mTOR, mammalian target of rapamycin; PE, phosphatidylethanolamine; ROS, reactive oxygen species; TSC1/2, tuberous sclerosis complex 1/2

Received 21.1.09; revised 20.2.09; accepted 24.2.09; Edited by M Piacentini; published online 27.3.09
} 


\section{Conceptual Cross-talk}

Three different types of interplay have come to light, each of which is equally valid for a particular cell type, stimulus and environment (Figure 1). Both apoptosis and autophagy can act as partners to induce cell death in a coordinated or cooperative manner (Figure 1a); autophagy acts as an antagonist to block apoptotic cell death by promoting cell survival (Figure 1b), or autophagy acts as enabler of apoptosis, participating in certain morphologic and cellular events that occur during apoptotic cell death, without leading to death in itself (Figure 1c).

The partner. In this relationship, both apoptosis and autophagy cooperate to lead to cell death. A corollary of this is that if one program is blocked (by genetic defect or pharmacological inhibition), the other takes over. In this scenario, the death programs serve as back-ups to ensure efficient cell death. They may occur simultaneously and even cooperatively, although one may predominate and therefore obscure evidence of the other. Or, alternatively, the second pathway may only be activated upon the failure of the first, implying that the first death pathway provides a means of blocking the second as long as it is functional. In either of these cases, the goal of both death programs is the same, that is, to eliminate the cell. The cooperativity and/or negative feedback imply that there is coordination between the pathways. This may occur at the activation level, or there may be a built-in molecular gauge to measure the efficiency of one so that the other knows to take over. a

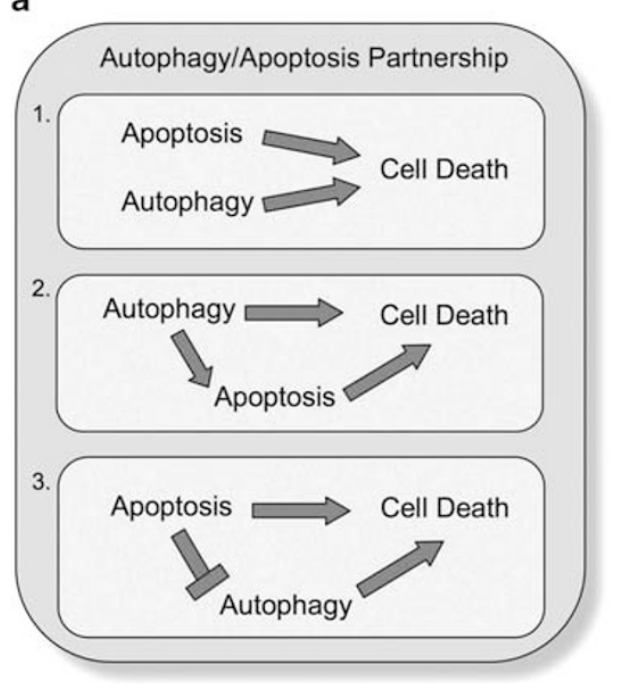

b

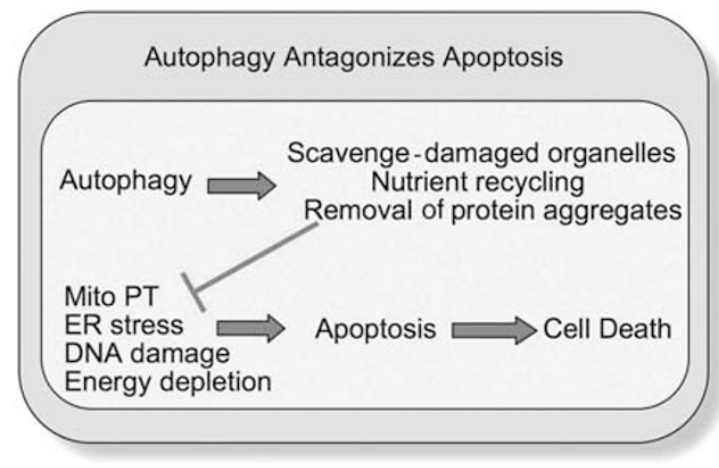

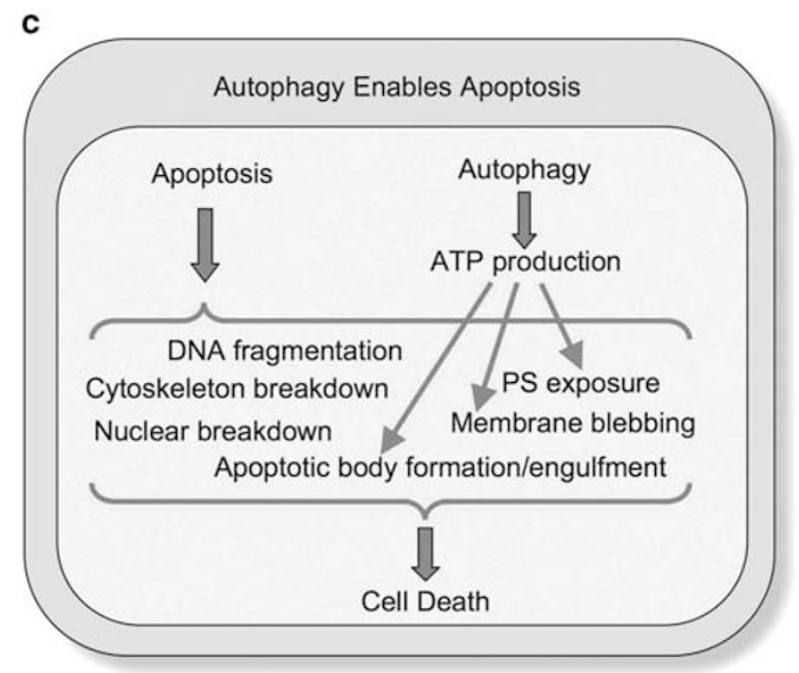

Figure 1 Schematic representation of the cross-talk between apoptosis and autophagy. (a) Autophagy and apoptosis are partners, both leading to cell death. They may act independently in parallel pathways (1), or one may influence the other. Autophagy has been shown to be necessary for apoptotic cell death, placing it upstream of apoptosis, whereas simultaneously modulating an independent means of death (2). Or, apoptosis may suppress autophagy, so that the latter is only evident on inhibition of apoptosis (3). (b) Autophagy antagonizes apoptotic cell death by promoting cell survival, through, for example, the removal of damaged organelles that are a source of genotoxic ROS, or by catabolizing cellular macromolecules to provide a source of nutrients and energy for the starved cell, or by limiting ER stress through the degradation of unfolded protein aggregates. These functions block the stimuli that would trigger an apoptotic response. (c) Autophagy, although not leading to cell death by itself, enables the apoptotic program by participating in certain morphological changes, such as ATP-dependent events such as PS exposure and membrane blebbing 
Numerous studies showing the cooperative relationship between apoptosis and autophagy exist in the literature. Several commonly known inducers of apoptosis have been shown to also activate autophagy, such as etoposide in mouse embryonic fibroblasts (MEFs) ${ }^{2}$ ceramide in breast and colon carcinoma ${ }^{3}$ and activation of the TRAIL receptor- 2 in cancer cells. ${ }^{4}$ Furthermore, the simultaneous activation of both pathways has been observed in experimental settings and in clinical trials. For example, arsenic trioxide treatment of T-lymphocytic leukemias activated both apoptosis and autophagy and both processes contributed to cell death and complete tumor remission. ${ }^{5}$ Both autophagy and apoptosis were likewise induced and necessary for cell death on treatment of Kaposi's sarcoma with imatinib, ${ }^{6}$ PC3 prostate cancer cells with the proteasome inhibitor MG132 ${ }^{7}$ and $\mathrm{HL}-60$ leukemia cells with vitamin $\mathrm{K} 2{ }^{8}$ In the last case, autophagy was especially prominent when apoptosis was blocked by the expression of $\mathrm{Bcl}-2$. Following endoplasmic reticulum (ER) stress, characteristics of both apoptosis and autophagy were present in the mouse kidney and in cultured MEFs, and suppression of both death programs through the knock down or knockout of apoptotic and autophagic genes was necessary to achieve a significant increase in cell survival. ${ }^{9}$

Furthermore, there are several reported cases in which autophagy actually enhances caspase-dependent cell death and is required for the latter. As a result, blocking autophagy prevents apoptosis. For example, autophagy and apoptosis are sequentially observed in models for HIV infection of CD4 ${ }^{+}$ $\mathrm{T}$ lymphocytes, utilizing the expression of HIV envelope glycoprotein on co-cultured cells or the expression of its cell surface receptor, CXCR4, on the target cell. ${ }^{10}$ Inhibition of autophagy by the knock down of Atg7 or Beclin 1, or treatment with 3-MA, suppressed caspase activation and attenuated cell death. In contrast, inhibition of apoptosis accelerated the induction of autophagy markers and only partially attenuated cell death. This implies that autophagy functions both upstream of apoptosis and independently leads to cell death. A similar relationship was observed in light-damaged retinas in the intact mouse or $\mathrm{H}_{2} \mathrm{O}_{2}$-treated photoreceptor cells, treatments that induce oxidative stress. ${ }^{11}$ Inhibition of either pathway partially rescued cell survival, and autophagy was again shown to be necessary for the induction of apoptosis. Interestingly, simultaneous inhibition of both pathways led to increased cell death by necrosis, which was not evident as long as one of the other death programs was functional. ${ }^{11}$ Inhibition of autophagy likewise prevented apoptosis following several other death signals, including TNF $\alpha$ treatment of sarcoma cells in combination with NF- $\mathrm{BB}$ suppression, ${ }^{12} \mathrm{ER}$ stress in non-transformed fibroblasts, ${ }^{13}$ and serum and potassium deprivation of cerebellar granule cells. ${ }^{14}$ In this last case, caspase-3 activation and apoptosis were dependent on the release of cathepsin B from an endosomal/ lysosomal compartment, suggesting a possible molecular link between autophagy and apoptosis.

Under other circumstances, autophagy is restrained by the apoptotic pathway and is evident only when the latter is inhibited. For example, autophagic cell death mediated by the receptor-interacting protein and c-jun $\mathrm{N}$-terminal kinase (JNK) was activated on inhibition of caspase-8. ${ }^{15}$ Cell death was attributed to the selective autophagic degradation of catalase, leading to the accumulation of reactive oxygen species (ROS) and mitochondrial outer membrane permeability (MOMP), which was inhibited by the knock down of Atg7 or Atg8. ${ }^{16}$ Similarly, etoposide, staurosporine or thapsigargin (an inducer of ER stress) activated autophagy in apoptosisincompetent Bax/Bak-/- MEFs, and cell viability was rescued by the inhibition of autophagy. ${ }^{17}$ Cell death was likewise blocked by Beclin 1 RNAi and chemical inhibitors of autophagy in macrophages treated with lipopolysaccharides and the caspase inhibitor zVAD, the combination of which resulted in autophagy. ${ }^{18}$

In all of the diverse situations described above, apoptosis, autophagy or both are used to eliminate the cell. The end result is the same for each pathway, in some cases achieving this end in a cooperative manner, and in some cases, in a mutually exclusive manner that acts as a back-up.

The antagonist. In this case, apoptosis and autophagy are distinct processes with disparate goals. Autophagy does not lead to cell death, but instead acts to attenuate apoptosis by creating a cellular milieu in which survival is favored. For example, in various settings of ER stress it has been shown that autophagy can maintain ER function through the consumption of protein aggregates and misfolded proteins, thus limiting the ER stress response and subsequent apoptosis. $^{13,19-22}$ Interestingly, in one case of ER stress in MEFs, autophagy promoted survival only in apoptosiscompetent cells; in Bax/Bak-/- cells, autophagy was associated with increased necrotic cell death. ${ }^{23}$ Autophagy can also serve to provide a source of energy and nutrients when these factors are limited, by catabolizing cellular organelles and macromolecules, thereby prolonging cell viability. Thus, autophagy was shown to be essential to survival during periods of starvation in adult mice ${ }^{24}$ and neonatal mice in the feeding adaptation period that immediately follows birth, ${ }^{25}$ and in several cell culture models of nutrient depletion. ${ }^{26,27}$ Autophagy was also shown to protect non-transformed epithelial cells from anoikis, the loss of matrix attachment that induces apoptotic cell death. ${ }^{28}$

Autophagy is also a mechanism to maintain genomic integrity in the face of metabolic stress, drug treatment or radiation damage, ${ }^{29-32}$ for example, by scavenging depolarized mitochondria that are a source for genotoxic ROS, a process known as mitophagy. ${ }^{33}$ Consistent with this, in the absence of autophagy, DNA damage, gene amplification and chromosomal abnormalities were evident following metabolic stress in tumor cells. ${ }^{29,30}$ Such overwhelming DNA damage would normally activate an apoptotic response. Accordingly, inhibition of autophagy in breast, prostate and colon cancer cells, and in malignant glioma cells enhanced the death response of these cells to radiotherapy. ${ }^{31,32}$ Likewise, inhibition of autophagy increased the antineoplastic potency of the histone deacetylase inhibitor SAHA in imatinib-resistant primary CML cells ${ }^{34}$ and the antiangiogenic effects of kringle 5 in endothelial cells, by activating apoptotic cell death. ${ }^{35}$ Furthermore, mitophagy may directly limit apoptosis by preventing MOMP and the subsequent release of pro-apoptotic molecules such as cytochrome $C$ and Smac/Diablo. ${ }^{33}$

Thus, the cell utilizes autophagy to promote survival in many circumstances. As a corollary, suppression of 
autophagy can promote apoptosis in these cell settings as a response to DNA damage, ER stress or loss of nutrient and growth factor signaling pathways.

The enabler. In this interplay, autophagy assists the apoptotic program without leading to death in itself, by enabling the execution of particular components of apoptotic cell death. For example, autophagy-dependent maintenance of cellular ATP levels during nutrient deprivation enables phosphatidylserine exposure, an ATP-dependent process. Furthermore, in vivo, phosphatidylserine exposure provides the 'eat me' signal to neighboring phagocytes and thus mediates cell engulfment and clearance of apoptotic bodies. In the absence of autophagy, or an external source of ATP, cell corpses persist during embryonic developmental apoptosis, indicating that autophagy is indirectly required for this stage as well. ${ }^{36}$ Membrane blebbing is also an energy-dependent event, which requires ATP-driven actomyosin contraction, and thus autophagy may participate in this process. ${ }^{37}$ This cross-talk may be mistaken for a causative role in apoptotic cell death (as in the section The Partner above), when in fact autophagy may only be necessary for a particular hallmark of apoptosis. Thus, cell death assays such as Annexin $\mathrm{V}$ binding that examine an ATP-dependent feature should be supplemented with other independent criteria. When autophagy acts as an enabler, its inhibition is predicted to prevent the ATP-dependent features only, without affecting other apoptotic responses, whereas according to the causative model, suppression of autophagy should prevent the entire set of molecular events characteristic of apoptosis.

\section{Molecular Cross-talk}

At the molecular level, the cross-talk between apoptosis and autophagy is manifested by the numerous genes that are shared by both pathways. These include regulatory genes such as $p 53$ and p19ARF, as well as some of the basic machinery that execute the death programs (e.g. Atg5, Bcl-2). Thus, stimuli that lead to the activation or suppression of these genes will affect both pathways.

The mammalian target of rapamycin signaling pathway. The cross-talk between apoptosis and autophagy can exist at the level of the signal-transduction pathways that sense death/survival signals and translate them to molecular events that activate or inhibit the specific death programs. Mammalian target of rapamycin (mTOR) and its regulators (Figure 2a) play a pivotal role as molecular sensors of cellular energy, growth factor and nutrient levels, and promote cell growth and protein synthesis, and the inhibition of autophagy, when these factors are abundant (reviewed in Corradetti and Guan ${ }^{38}$ Guertin and Sabatini ${ }^{39}$ ). When they are scarce, mTOR is inactivated and autophagy is induced as a compensatory mechanism. This is mediated, at least in yeast, by the Atg1 kinase and its positive regulator Atg13. When active, yeast TOR phosphorylates Atg13, preventing its association with Atg1 (mammalian Ulk1/2), thereby blocking Atg1's function, which is critical for autophagosome formation. mTOR activity is regulated by

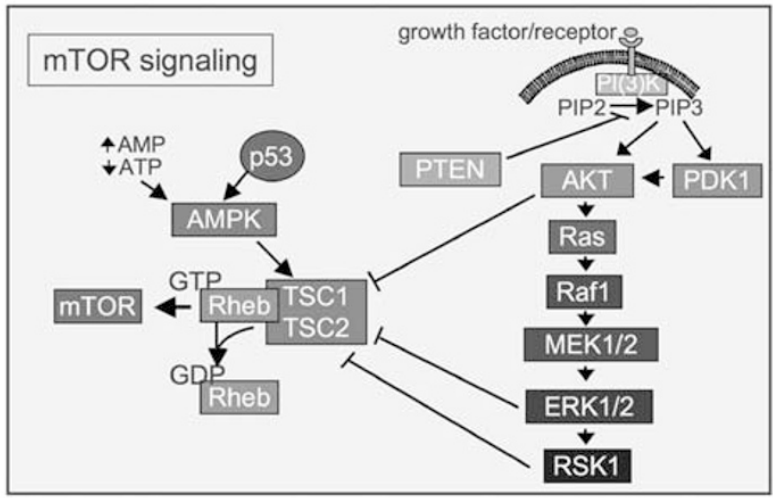

b

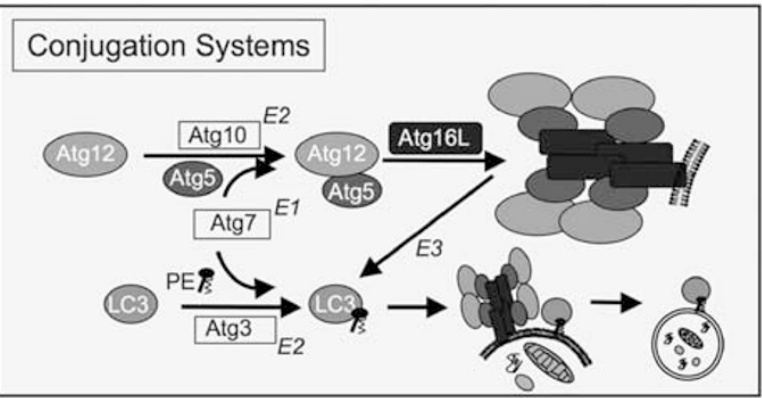

Figure 2 Autophagic signaling events. (a) The mTOR pathway and its regulators. Through modulation of TSC1/TSC2, mTOR is activated by growth factor signaling and inactivated by elevated AMP/ATP ratios. (b) The two ubiquitin-like conjugating systems are necessary for the elongation of the autophagosome membrane. The Atg5-Atg12 dimer facilitates LC3-PE conjugation and the multimeric complex with $A t g 16 \mathrm{~L}$ determines its site of tethering to the initiation membrane, before the complex is released as the autophagosome matures

Rheb, a small GTP-binding protein that activates mTOR in its GTP-bound form. GTP hydrolysis, promoted by the tuberous sclerosis complex 1/2 (TSC1/TSC2) dimer, inactivates Rheb, thus negatively regulating mTOR activity. Numerous signaling molecules regulate mTOR activity through modulation of these regulatory factors. For example, Akt, ERK or RSK, all of which are activated by growth factors of the class I PI(3)K pathway, phosphorylate and inactivate TSC1/TSC2, thus enabling Rheb activation of mTOR and consequent inhibition of autophagy (reviewed in Corradetti and Guan ${ }^{38}$ Guertin and Sabatini ${ }^{39}$ ). Conversely, AMP-activated kinase (AMPK), which is activated by high AMP/ATP ratios present when nutrients are limited, induces autophagy through a phosphorylation event that stimulates TSC1/TSC2 activity (reviewed in Wouters and Koritzinsky ${ }^{40}$ ). The signaling molecules described here are by no means specific to autophagy and have a wide range of activities within the cell, in particular in the context of cell survival or death. Many, such as Akt and ERK, activate pathways that also affect apoptosis (reviewed in Song et al., ${ }^{41}$ McCubrey et al. ${ }^{42}$ and Ballif and Blenis ${ }^{43}$ ). For example, Akt- or RSK2-mediated phosphorylation of Bad, a BH3-only member of the Bcl-2 family, leads to its dissociation from $\mathrm{Bcl}-2$, freeing $\mathrm{Bcl}-2$ to inhibit Bax/Bak-mediated apoptosis. Additionally, Akt antagonizes the transcriptional activity of the FOXO family of transcription factors by direct phosphorylation, and also p53, by the phosphorylation of Mdm2, resulting in reduced expression of 
several pro-apoptotic genes. Conversely, phosphorylation of CREB by either ERK-activated RSK1 or Akt activates its transcriptional activity toward antiapoptotic genes such as $B c l-2 / X_{L}$. Akt also negatively regulates several kinases that lie upstream of JNK, thereby inhibiting JNK activation and its pro-apoptotic functions.

Atg5. Atg5 is one of the components of the basic autophagic machinery (Figure 2b; reviewed in Gozuacik and Kimchi ${ }^{44}$ ). It participates in the two ubiquitin-like conjugation systems that are essential for elongation of the autophagosome membrane during vacuole formation. In the first step, Atg5 is covalently conjugated to Atg12, a ubiquitin-like protein, a reaction facilitated by the $\mathrm{E} 1$ and E2-like proteins Atg7 and Atg10, respectively. The Atg12-Atg5 dimer then binds Atg16L, forming a large multi-protein complex, which is recruited to the forming autophagosome's isolation membrane through Atg16L. In the second conjugation system, Atg7 and Atg3 similarly act as E1 and E2 enzymes mediating the conjugation of Atg8 (mammalian LC3) to the lipid phosphatidylethanolamine (PE). The Atg12-Atg5 dimer has E3-like activity for Atg8 conjugation to $\mathrm{PE}^{45}$ and the site of LC3 lipidation is determined by the membrane localization of the Atg16 $\mathrm{L}$ complex $^{46}$ (see Figure 2b). Lipidation of Atg8, which occurs only under stimulation of autophagy, converts Atg8 from its soluble cytoplasmic form (LC3-I) to the membrane-bound, autophagosome-associated, form (LC3-II), which is required for membrane expansion.

Atg5, in addition to its essential role in autophagy, can also influence apoptotic signaling pathways. Following various apoptotic stimuli, Atg5 is cleaved by calpain, forming an $\mathrm{N}$-terminal product that translocates to the mitochondria, interacts with $\mathrm{Bcl}-\mathrm{X}_{\mathrm{L}}$ and promotes cytochrome $C$ release and caspase activation. ${ }^{47}$ Thus, Atg5 is appropriated away from the autophagy pathway and recruited to the apoptotic signaling pathway. Furthermore, one can assume that this cross-talk is a mechanism by which apoptosis suppresses autophagy, perhaps under circumstances when the latter's pro-survival functions would counter cell death.

Bcl-2 proteins. Bcl-2 is probably the most well-known inhibitor of cell death; its isolation as an oncogene was the first indication that inhibition of cell death can lead to tumorigenesis. ${ }^{48}$ The cytoprotective function of $\mathrm{Bcl}-2$ stems from its ability to antagonize Bax/Bak, block MOMP, and thus prevent apoptosis. More recently, Bcl-2 was also shown to inhibit autophagy, ${ }^{49}$ whereas several other family members or modulators activate autophagy (reviewed in Levine et $a .^{50}$ ). These regulate autophagy through the Vps34 kinase complex, a class III $\mathrm{PI}(3) \mathrm{K}$ that is necessary for autophagosome formation. The complex contains multiple proteins that are essential for kinase activity, including Beclin 1 (the mammalian homolog of Atg6), UVRAG, Ambra1 and Bif-1 (Figure 3). Bif-1, a member of the endophilin B family, also regulates apoptosis by binding and/or activating Bax/Bak. ${ }^{51}$ Beclin 1 binds $\mathrm{Bcl}-2 / \mathrm{Bcl}-\mathrm{X}_{\mathrm{L}}$ through a $\mathrm{BH} 3$ domain that mediates docking to the latter's BH3-binding groove. The binding of $\mathrm{Bcl}-\mathrm{X}_{\mathrm{L}}$ to Beclin 1 interferes with Beclin 1/Vps34 complex formation by reducing Beclin 1's monomerization and interaction with UVRAG. ${ }^{52}$ The constitutive $\mathrm{Bcl}-2 / \mathrm{Bcl}-\mathrm{X}_{\mathrm{L}}-$
Beclin 1 association is disrupted by signals that promote autophagy. For example, Beclin 1 is phosphorylated by death-associated protein kinase (DAPk) (see below) in the $\mathrm{BH} 3$ domain, which reduces its affinity for $\mathrm{Bcl}-\mathrm{X}_{\mathrm{L}}{ }^{53}$ In the reciprocal manner, JNK phosphorylates $\mathrm{Bcl}-2$, triggering its release from Beclin 1, ${ }^{54}$ following starvation or ceramideinduced autophagy., ${ }^{3,54}$ A second mechanism leading to dissociation of the complex involves the competitive displacement of Beclin 1's BH3 domain from Bcl-2/Bcl- $X_{L}$ by other $\mathrm{BH} 3$-containing proteins, ${ }^{50}$ such as BNIP3 ${ }^{55}$ BNIP3 is a $\mathrm{BH}$-only member of the $\mathrm{Bcl}-2$ family that is upregulated by hypoxia and previously reported to play a role in apoptosis. ${ }^{56}$ It should also be noted that Beclin 1 and Vps34 are not always essential for autophagy; a non-canonical autophagic pathway, occurring independently of Beclin 1 and Vps34, was demonstrated in neurons treated with the neurotoxin 1-methyl-4-phenylpyridinium ${ }^{57}$ and in breast cancer cells treated with the polyphenol resveratrol. ${ }^{58}$ In both cases, the non-canonical autophagy induced cell death. Interestingly, Bcl-2, which antagonizes canonical autophagy, had a stimulatory effect on resveratrol-induced autophagy. ${ }^{59}$

It is not clear how the apoptotic versus autophagic functions of the Bcl-2 family members are determined. A simple explanation is that the different functions are spatially separated and determined by the proteins' localizations at the mitochondria or ER. For example, ER-localized Bcl-2, but not the mitochondrial pool, is regulated by JNK phosphorylation. Mitochondrial pools of ceramide, on the other hand, can lead to the dephosphorylation of $\mathrm{Bcl}-2$ by protein phosphatase $2 \mathrm{~A}$, thereby activating apoptosis. ${ }^{60}$

p53. p53 is a known activator of apoptosis, responsible for upregulating pro-apoptotic genes at the transcriptional level, such as Bax, PUMA and NOXA, and inhibiting the expression of antiapoptotic ones, such as $\mathrm{Bcl}-2$ (see Fridman and Lowe ${ }^{61}$ for review). Cytoplasmic p53 also enhances Bax-induced MOMP. More recent reports suggest that p53 positively regulates autophagy as well (Figure 3). One mechanism proposed involves the inhibition of the mTOR pathway following activation of p53 by the DNA-damaging agent etoposide. mTOR inhibition was dependent on AMPK activation by $\mathrm{p} 53$ and required TSC1/TSC2. ${ }^{2}$ Transcriptional activation of damage-regulated modulator of autophagy (DRAM), a lysosomal membrane protein that is upregulated by p53 following DNA damage, is a second means by which p53 can induce autophagy. ${ }^{62}$ Interestingly, DRAM expression was required for p53-induced autophagy and subsequent cell death, as well as apoptotic cell death from DNA-damaging agents. Thus, signals that activate p53 can lead to both apoptosis and autophagy.

Interestingly, p53 may play a dual role in autophagy regulation. In contrast to the positive effects on autophagy described above, loss of p53 function (through genetic manipulation or pharmacological inhibition) is in itself sufficient to trigger full activation of autophagy. ${ }^{63}$ The antiautophagic property was attributed to cytoplasmic p53. Several autophagic stimuli induce HDM2-dependent degradation of $\mathrm{p} 53$, enabling autophagy by eliminating the restrictive p53. ${ }^{63}$ Note that the autophagy described here is not associated with cell death, but rather promotes cell survival. For example, 


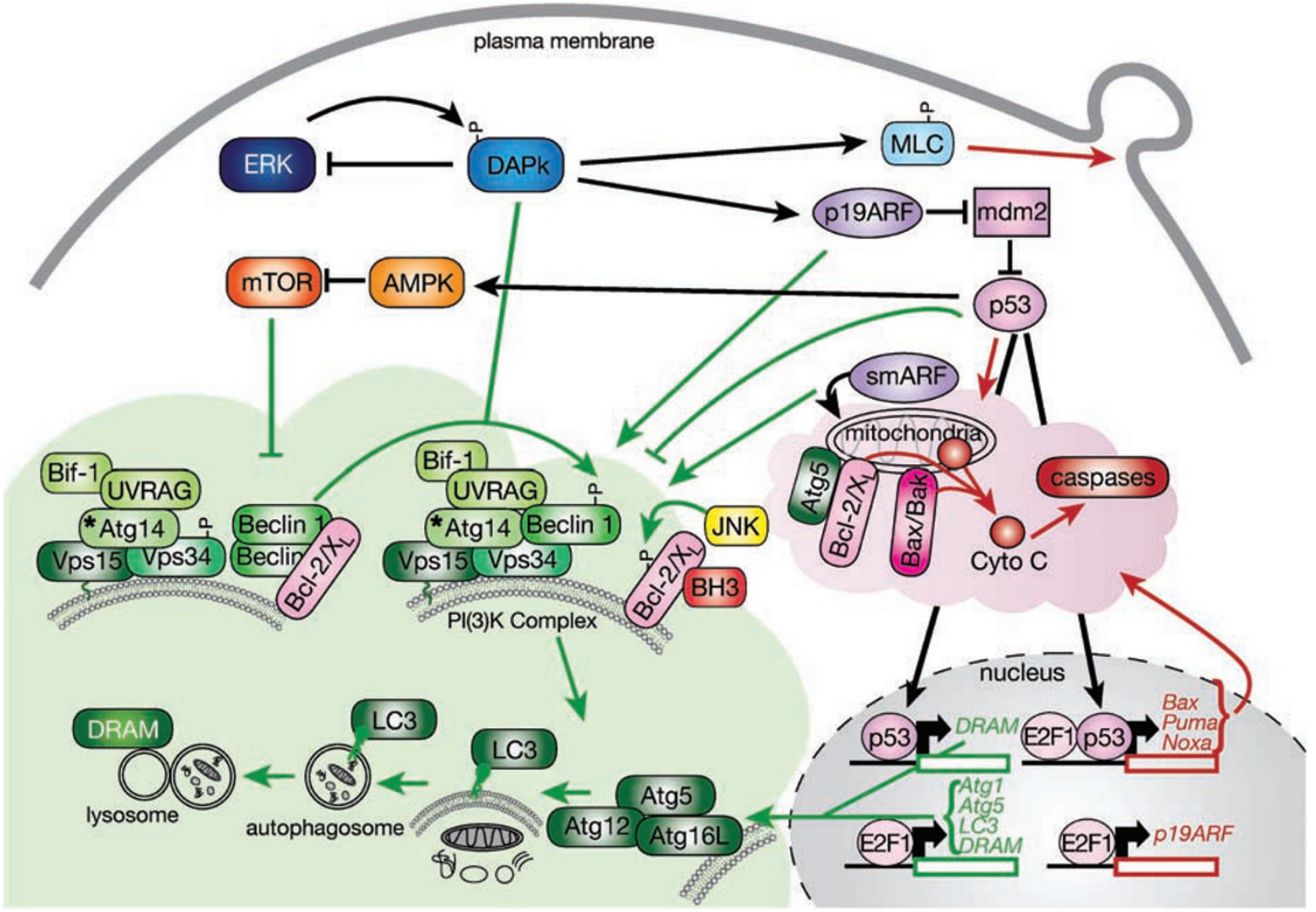

Figure 3 Common molecular regulators: genes that affect both autophagy and apoptosis and the pathways that they influence. Green-shaded area and green arrows indicate autophagic program, whereas red shading and arrows indicate apoptosis. Black arrows are upstream signaling events, and various cellular compartments are labeled as such. Phosphorylation events are indicated by P. *It is noted that although both UVRAG and mammalian Atg14 have been shown to bind Beclin 1 and to be essential for autophagosome formation, the exact nature of the complex on the isolation membrane is controversial, and in contrast to the simplistic presentation here, UVRAG and Atg 14 may define two distinct Beclin 1/Nps34 complexes

cancer cells lacking p53 utilize the enhanced autophagy to maintain ATP levels in conditions of nutrient and energy deprivation. Thus, regulation of autophagy by p53 is dependent on p53 localization; nuclear p53 favors induction of autophagy, leading to cell death, whereas cytoplasmic accumulation represses basal pro-survival autophagy. Loss of p53 thus provides two levels of growth advantage to tumor cells; it removes two mechanisms of eliminating the cell in response to genotoxic stress, and at the same time, enables cell survival under limiting nutrient conditions. Paradoxically, each of these exploits the same process, autophagy, utilizing its opposing functions.

ARF. The ARF tumor suppressor protein ( $\mathrm{p} 14^{\mathrm{ARF}}$ in human and $\mathrm{p} 19^{\mathrm{ARF}}$ in mouse), a product of the INK4A/ARF locus, has also been linked to both apoptosis and autophagic cell death (Figure 3). p19ARF is an upstream activator of p53, leading to the latter's protein stabilization by antagonizing p53's negative regulator Mdm2. As such, it functions in the p53 regulatory pathway to induce apoptotic genes and/or autophagy. The p19ARF mRNA can also produce a second, small isoform, known as smARF, which is generated by internal translation. ${ }^{64}$ smARF lacks the $\mathrm{N}$-terminal domains that mediate nuclear localization and Mdm2 binding, and instead localizes to the mitochondria, induces mitochondrial depolarization and autophagic cell death. Within a proteinase K-resistant compartment of the mitochondria, smARF interacts with the p32 mitochondrial protein, which stabilizes smARF and as a consequence, increases its ability to cause mitochondrial membrane dissipation and autophagy. ${ }^{65}$ Fulllength p19ARF and its $\mathrm{N}$-terminal domain were also shown to induce autophagy on overexpression in p53-containing cells and in p53-deficient cells, the latter indicating a mechanism that does not involve the p53-mediated autophagy described above. ${ }^{66}$ Yet, a further detailed study established that nucleolar localized full-length p19ARF, unlike the mitochondrial smARF, is incapable of inducing p53-independent autophagy. ${ }^{67}$ An additional study suggested that human p14ARF also localizes to the outer membrane of mitochondria where it binds $\mathrm{Bcl}-2 / \mathrm{X}_{\mathrm{L}}$, thereby reducing the latter's association with Beclin 1, ${ }^{68}$ although it is unclear how an interaction at the mitochondria affects the ER-localized $\mathrm{Bcl}-2 /$ Beclin 1 complex. Oncogene expression results in the induction of both full-length p19ARF and smARF, ${ }^{64}$ suggesting that smARF's contribution to autophagy is more prominent upon specific cell signals.

DAPk. One of the most striking among the commonly shared genes is $D A P k$, a $\mathrm{Ca}^{2+} /$ calmodulin-regulated Ser/ Thr kinase that associates with the cytoskeleton. ${ }^{69} \mathrm{DAPk}$ is a 
tumor suppressor, functioning as part of an early p53dependent transformation checkpoint and also as an inhibitor of metastasis. ${ }^{69}$ Although the antimetastatic properties can be attributed directly to DAPk's effects on cell motility, ${ }^{70,71}$ most of its tumor-suppressive nature stems from its ability to modulate both apoptosis and autophagy (Figure 3). DAPk activity was shown to be necessary for apoptotic cell death induced by death receptor activation, hyperproliferation signals generated by forced oncogene expression, anoikis induced by loss of adhesion, ceramide and TGF $\beta .^{69}$ Furthermore, DAPk activity is also required for autophagic cell death, such as that induced by IFN $\gamma$ in HeLa cells, ${ }^{72}$ and mutation or knock down of the Caenorhabditis elegans DAPk ortholog attenuated starvation-induced autophagy in the pharyngeal muscle. ${ }^{73}$ Interestingly, tunicamycin-induced ER stress resulted in both apoptosis and autophagy in MEFs, both of which were reduced in DAPk-/- cells. ${ }^{9}$ Thus, in this system, DAPk regulates both death processes simultaneously, suggesting a critical role for DAPk as an integrator of apoptotic/autophagic cross-talk. Furthermore, ectopic expression of DAPk has been shown to induce autophagic vesicle formation and membrane blebbing, the latter a characteristic observed in cells dying by either apoptosis or autophagy. ${ }^{37}$

DAPk's multiple roles in cell death are likely mediated by the phosphorylation of multiple substrates, although only a limited number of such substrates have been identified ${ }^{69}$ (Figure 3). Its ability to induce membrane blebbing and influence stress fiber formation stems from its ability to phosphorylate myosin II regulatory light chain (MLC). ${ }^{74,75}$ As mentioned above, DAPk phosphorylates Beclin 1, thereby reducing the latter's binding to $\mathrm{Bcl}-\mathrm{X}_{\mathrm{L}}$, presenting a mechanism by which it may induce autophagy. ${ }^{53}$ The phosphorylation was mapped to a single site (T119) within Beclin 1's BH3 domain and was shown to be critical for the dissociation of this important complex. ${ }^{53}$ DAPk has also recently been shown to interact with MAP1B, ${ }^{76}$ which can bind LC3 and associate with autophagosomes on phosphorylation. ${ }^{77}$ In addition, DAPk influences overall cell survival by modulating several signaling molecules. DAPk upregulates p53 through a mechanism that requires p19ARF. ${ }^{78}$ As p53 can activate either apoptosis or autophagy, this potentially links DAPk to both death pathways, in addition to its p53-independent effects on autophagy. ${ }^{37}$ ERK, which phosphorylates and activates DAPk, is in turn regulated by DAPk, which promotes its retention in the cytoplasm. This results in the inhibition of ERK's nuclear functions, including pro-survival signaling that counter apoptosis. ${ }^{79}$ DAPk has also recently been shown to phosphorylate PKD, which leads to the activation of JNK and necrotic cell death during oxidative stress. ${ }^{80}$

E2F1. The E2F family of transcription factors has been implicated in various signaling pathways leading to cell death and survival. E2F1 triggers apoptosis by upregulating p53 through p19ARF, an E2F1 target gene, and by enhancing p53-mediated transactivation of pro-apoptotic genes. ${ }^{81}$ More recently, E2F1 was shown to induce autophagy by upregulating the expression of the autophagy genes LC3, Atg1, Atg5 and DRAM. ${ }^{82}$ Thus, E2F1 is a molecular link between genes necessary for apoptosis and genes involved in autophagy.

\section{Cross-talk in Cancer}

The complex interplay between apoptosis and autophagy is not only of theoretical significance to the researcher, but will impact on the course of treatment for disease that is associated with cell death. Clinical investigators have long exploited cell death mechanisms in the treatment of cancer, on recognition that malignancies result from an increase in cell number due to the disruption of the delicate balance between cell proliferation and elimination. In most cases, stimulation and/or restoration of apoptotic cell death leads to suppression of transformation and tumorigenesis. The contribution of autophagy to tumorigenesis is less obvious, and one must carefully consider the paradoxical nature of autophagy and its effects on apoptosis before utilizing chemo-therapeutic strategies to modulate autophagy. The three types of autophagy must be considered: basal housekeeping autophagy important for protein and organelle turnover, starvation and stress-induced autophagy that promotes cell survival, and autophagy that exceeds the safe threshold and leads to cell death. These multiple tasks can account for the fact that in a clinical setting, inhibition of autophagy has been associated both with increased tumor growth and tumor regression. Therefore, an analysis of the various steps in the process from normal cell to transformed cell to malignant tumor and the potential role that autophagy can play at each stage is necessary. It should be noted that the experimental evidence is still not sufficient to decisively construe the exact role that autophagy and its interplay with apoptosis has in cancer progression; here, we present a model that can explain the current data (Figure 4).

(1) Precancerous stages: The cell is exposed to a mutagen, pathogen or other cellular stress that causes DNA damage, mitochondrial damage and the release of ROS, resulting in the accumulation of gene mutations that disrupt cell cycle control, DNA repair or cell death. At this stage, the homeostatic function of autophagy to eliminate damaged organelles (e.g. mitophagy) and maintain genomic stability can block or limit the cumulative effects of the cellular stress that promote cellular transformation..$^{29,30,83}$ Suppression of autophagy at this stage would accelerate tumor development. This is especially true if mechanisms facilitating cell cycle arrest and/or apoptosis are also defective. Consistent with this, gene amplification, DNA damage and aneuploidy were prominent in Beclin 1 heterozygote cells that overexpressed $\mathrm{Bcl}-2$, which cannot undergo apoptosis. ${ }^{30}$

(2) The transformed cell: If the DNA damage is extensive and cannot be contained by autophagy, the cell can still avoid transformation by initiating a death response. As the cancer cell proliferates, loses its anchorage-dependent growth and migrates away from its source, it will encounter additional stimuli that can trigger cell death. The cell can die by either apoptosis or by autophagy that surpasses the safe threshold. The presence of a back-up death mechanism ensures cell elimination even if one pathway has been disabled by gene mutation. Suppression of autophagy or apoptosis at this stage would again accelerate tumor development. 


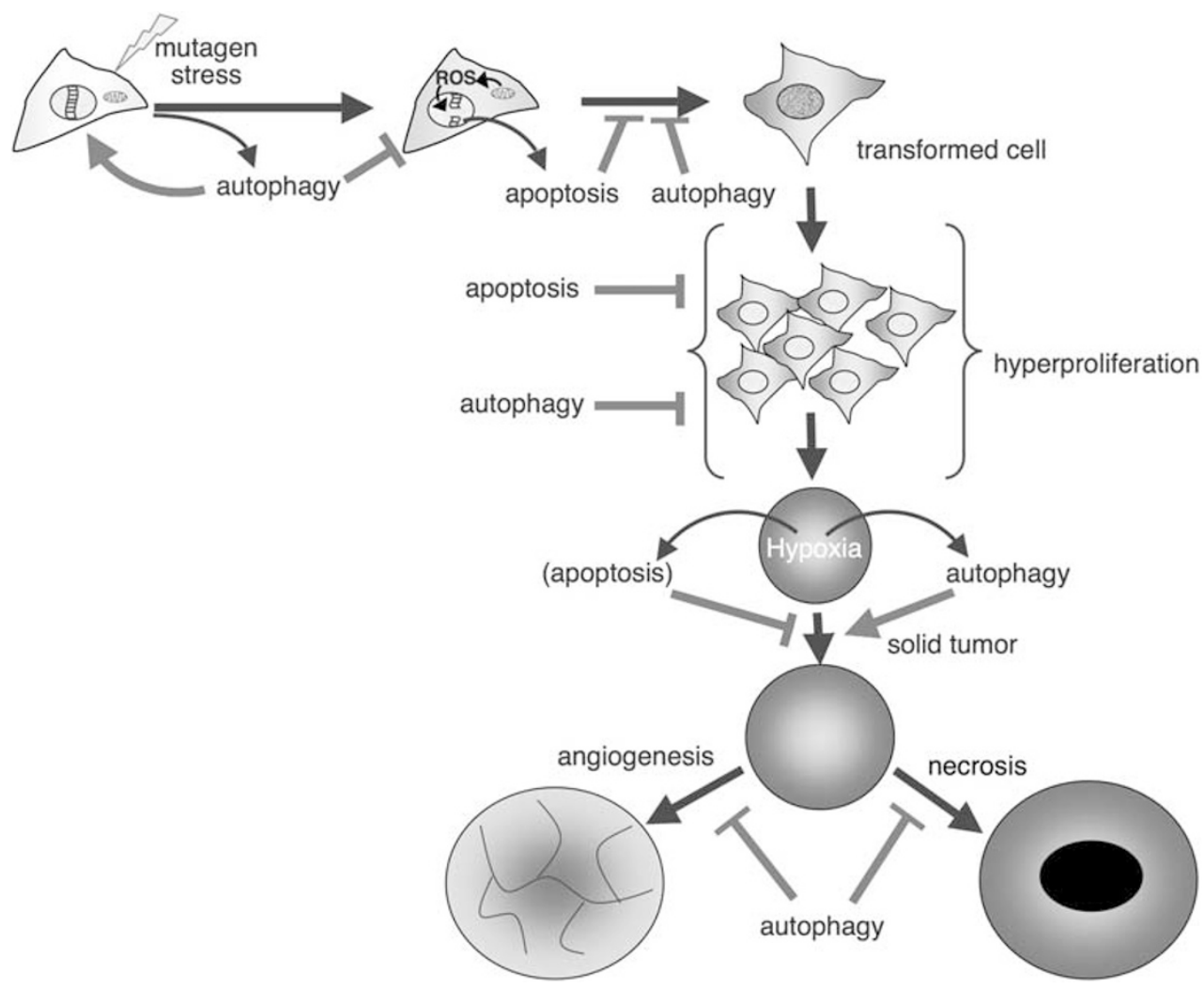

Figure 4 The roles of autophagy and apoptosis in cancer development. Various stages from the precancerous cell that accumulates DNA damage to the transformed cell that undergoes uncontrolled proliferation to the solid tumor are shown. Although apoptosis leads to the elimination of the cell/tumor, autophagy can serve to either promote cell/ tumor survival at certain stages, or to promote its elimination, at other stages

(3) The solid tumor. The cancer cell is no longer an individualized unit, but can be considered part of an organ, the solid tumor. Solid tumors that are not (yet) vascularized, especially the tumor centers, develop regions of hypoxia and limited nutrient availability, creating a microenvironment of metabolic stress. Although this can trigger apoptotic cell death, such an environment selects for cells with defective apoptotic programs, such as loss of p53, ${ }^{84}$ and it is likely that by this point, the tumor cells will have lost the means of dying by apoptosis. Starvationactivated autophagy, on the other hand, acts to promote cell survival, by breaking down cellular building blocks to provide the missing nutrients. ${ }^{29,85}$ One would predict then that suppression of autophagy at this stage would inhibit tumor development. However, in actuality, in the absence of apoptosis, the autophagic program is antitumorigenic, because, as in the precancerous stages, it can limit the extent of further genomic damage that can lead to even more aggressive tumors. This was evident in vivo, in which allelic deletion of Beclin 1, and decreased autophagy, resulted in gene amplification in mouse mammary tumors in response to metabolic or replication stress. ${ }^{29}$ Furthermore, in the absence of both apoptosis and autophagy, necrotic cell death ensues, resulting in an inflammatory response involving macrophage infiltration, which can be associated with more aggressive tumor growth. ${ }^{85}$ At some point, the tumor adapts by promoting angiogenesis, enabling the supply of nutrients and oxygen and avoiding metabolic stress. Autophagy plays a role here too, as it has been shown to block angiogenesis. For example, the combination of caspase inhibition, irradiation and inhibition of mTOR, which stimulated autophagy, correlated with the suppression of angiogenesis and reduced tumor volume in vivo in a mouse model of lung cancer. ${ }^{86}$ Similarly, mTOR inhibition sensitized endothelial cells to radiation therapy, limiting vasculature development and blood flow in mouse glioma xenografts. ${ }^{87}$ Autophagy can also be antiangiogenic by the selective autophagic consumption of neuropilin-1, a receptor for VEGF on endothelial and carcinoma cells, following hypoxia and nutrient deprivation. ${ }^{88}$ Thus, although for the short term, autophagy can compensate for the limited nutrient supply, in the long run, it will inhibit the tumor's access to a blood supply, and impede tumor growth. For all these reasons, suppression of autophagy would indirectly accelerate tumor development.

The strongest evidence for the role of apoptosis or autophagy in the development of the tumor cell comes from the tumor itself: the genes that are inactivated or induced that provide a selective growth advantage. It has long been recognized that antiapoptotic genes such as $\mathrm{Bcl}-2$ are oncogenes, whereas those that promote apoptosis, such as p53, are tumor suppressors. Autophagic genes are also modulated in tumor cells, and can be classified as follows. (a) Oncogenic signaling genes such as ERK, Akt, RSK1 and 
$P I(3) K$ (class I) have been shown to suppress autophagy. Their contributions to cell survival are pleiotropic, however, and thus their status as oncogenes cannot solely be attributed to their effects on autophagy. (b) Oncogenes that more directly suppress autophagy such as Bcl-2. Notably, the oncogenic function of overexpressed, misregulated Bcl-2 can stem from its ability to block apoptosis, autophagy or both. (c) Tumor suppressors that activate autophagy, including inhibitors of the mTOR pathway, such as TSC1/TSC2, PTEN (a PIP3 phosphatase that antagonizes PI(3)K/Akt signaling), and p53 (through AMPK). Other tumor suppressors play a more direct role in regulating autophagy such as DAPk, ${ }^{89}$ Beclin 1, p19ARF (smARF) and UVRAG. ${ }^{90-93}$ p53 falls into this category as well through upregulation of DRAM, which has also been shown to be downregulated in certain carcinomas. ${ }^{62}$ Even these proteins, however, may exert their tumor suppressive activity through additional functions. p53 and p19ARF have multiple effects on cell survival, cell cycle progression and protein translation. DAPk also inhibits cell migration, thus providing another mechanism by which it blocks metastasis. ${ }^{71}$ Both Beclin 1 and UVRAG have been found to localize to endosomal membranes, and UVRAG especially may have a more general role in membrane fusion events than those needed exclusively for autophagosome maturation. ${ }^{94,95}$ Many of these proteins also influence apoptosis, again stressing the prominence of the cross-talk between the two pathways.

With the exception of Beclin 1, none of the Atg genes have been shown, so far, to be tumor suppressors or oncogenes. Does this imply that the Atg machinery is not important to the development of cancer, and the contribution of the genes listed above are due solely to their non-autophagic functions? As the Atg genes have been initially extensively studied in yeast, and their functions then extended to mammals, the lack of data on their contribution to tumorigenesis may simply be attributed to the fact that this issue has not yet been examined in detail. At this point, much of the focus is on understanding the molecular events that comprise each stage in autophagy; perhaps future research will be geared toward examining the presence/absence/mutational changes of these genes in various cancers. In addition, it is conceivable that the housekeeping functions of the basic machinery of autophagy would counteract any possible advantage that may be conferred to the tumor cells by loss of autophagy. Notably, among the apoptotic genes, it is also the regulators such as $p 53$ and $\mathrm{Bcl}-2$ that have been linked to cancer, and not the effector caspases. Therefore, it is intriguing to conclude that an oncogene/tumor suppressor may more likely be a regulator gene of autophagy than a component of the basic autophagic machinery.

In conclusion, the cross-talk between apoptosis and autophagy manifests itself in several layers. Autophagy itself presents a paradox as it can have opposing effects on cell survival and death, and as a result, its connection to apoptosis is complex. A full understanding of this multifaceted relationship will be critical for the assessment of anticancer strategies.

Acknowledgements. This study was supported by the European Commission (APO-SYS) and by the Israel Science Foundation (ISF). A Kimchi is the incumbent of Helena Rubinstein Chair of Cancer Research.
1. Kroemer G, Levine B. Autophagic cell death: the story of a misnomer. Nat Rev Mol Cell Biol 2008; 9: 1004-1010

2. Feng $\mathrm{Z}$, Zhang $\mathrm{H}$, Levine $\mathrm{AJ}$, Jin $\mathrm{S}$. The coordinate regulation of the $\mathrm{p} 53$ and $\mathrm{mTOR}$ pathways in cells. Proc Natl Acad Sci USA 2005; 102: 8204-8209.

3. Pattingre S, Bauvy C, Carpentier S, Levade T, Levine B, Codogno P. Role of JNK1dependent Bcl-2 phosphorylation in ceramide induced macroautophagy. J. Biol Chem 2008; 284: 2719-2728

4. Park K, Lee S, Kim T, Lee H, Lee C, Kim E et al. A human scFv antibody against TRAIL receptor 2 induces autophagic cell death in both TRAIL-sensitive and TRAIL-resistant cancer cells. Cancer Res 2007; 67: 7327-7334.

5. Qian W, Liu J, Jin J, Ni W, Xu W. Arsenic trioxide induces not only apoptosis but also autophagic cell death in leukemia cell lines via up-regulation of Beclin-1. Leuk Res 2007; 31: 329-339.

6. Basciani S, Vona R, Matarrese P, Ascione B, Mariani S, Cauda R et al. Imatinib interferes with survival of multi drug resistant Kaposi's sarcoma cells. FEBS Lett 2007; 581: 5897-5903.

7. Yang W, Monroe J, Zhang Y, George D, Bremer E, Li H. Proteasome inhibition induces both pro- and anti-cell death pathways in prostate cancer cells. Cancer Lett 2006; 243: 217-227.

8. Yokoyama T, Miyazawa K, Naito M, Toyotake J, Tauchi T, Itoh M et al. Vitamin K2 induces autophagy and apoptosis simultaneously in leukemia cells. Autophagy 2008; 4: 629-640.

9. Gozuacik D, Bialik S, Raveh T, Shohat G, Mizushima N, Yoshimori T et al. DAPk is a key mediator of endoplasmic reticulum stress-induced caspase activation and autophagy. Cell Death Diff 2008; 15: 1875-1886.

10. Espert L, Denizot M, Grimaldi M, Robert-Hebmann V, Gay B, Varbanov M et al. Autophagy is involved in T cell death after binding of HIV envelope proteins to CXCR4. J Clin Invest 2006; 116: 2161.

11. Kunchithapautham K, Rohrer B. Apoptosis and autophagy in photoreceptors exposed to oxidative stress. Autophagy 2007; 3: 433-441.

12. Djavaheri-Mergny M, Amelotti M, Mathieu J, Besancon F, Bauvy C, Souquere S et al. NFkappaB activation represses tumor necrosis factor-alpha-induced autophagy. J Biol Chem 2006; 281: 30373-30382

13. Ding WX, Ni HM, Gao W, Hou YF, Melan MA, Chen $X$ et al. Differential effects of endoplasmic reticulum stress-induced autophagy on cell survival. J Biol Chem 2007; 282 4702-4710.

14. Xue L, Fletcher G, Tolkovsky A. Autophagy is activated by apoptotic signalling in sympathetic neurons: an alternative mechanism of death execution. Mol Cell Neurosci 1999; 14: 180-198.

15. Yu L, Alva A, Su H, Dutt P, Freundt E, Welsh $S$ et al. Regulation of an ATG7-beclin 1 program of autophagic cell death by caspase-8. Science 2004; 304: 1500-1502.

16. Yu L, Wan F, Dutta S, Welsh S, Liu Z, Freundt E et al. Autophagic programmed cell death by selective catalase degradation. Proc Natl Acad Sci USA 2006; 103: 4952-4957.

17. Shimizu S, Kanaseki T, Mizushima N, Mizuta T, Arakawa-Kobayashi S, Thompson CB et al. Role of Bcl-2 family proteins in a non-apoptotic programmed cell death dependent on autophagy genes. Nat Cell Biol 2004; 6: 1221-1228.

18. Xu Y, Kim SO, Li Y, Han J. Autophagy contributes to caspase-independent macrophage cell death. J Biol Chem 2006; 281: 19179-19187.

19. Kouroku Y, Fujita E, Tanida I, Ueno T, Isoai A, Kumagai $H$ et al. ER stress (PERK elF2alpha phosphorylation) mediates the polyglutamine-induced LC3 conversion, an essential step for autophagy formation. Cell Death Differ 2007; 14: 230-239.

20. Ogata M, Hino S, Saito A, Morikawa K, Kondo S, Kanemoto S et al. Autophagy is activated for cell survival after endoplasmic reticulum stress. Mol Cell Biol 2006; 26 9220-9231.

21. Bernales S, McDonald K, Walter P. Autophagy counterbalances endoplasmic reticulum expansion during the unfolded protein response. PLOS Biol 2006; 4: e423.

22. Castino R, Davies J, Beaucourt S, Isidoro C, Murphy D. Autophagy is a prosurvival mechanism in cells expressing an autosomal dominant familial neurohypophyseal diabetes insipidus mutant vasopressin transgene. FASEB J 2005; 19: 1021-1023.

23. Ullman E, Fan Y, Stawowczyk M, Chen H, Yue Z, Zong W. Autophagy promotes necrosis in apoptosis-deficient cells in response to ER stress. Cell Death Differ 2007; 15 422-425.

24. Komatsu M, Waguri S, Ueno T, Iwata J, Murata S, Tanida I et al. Impairment of starvationinduced and constitutive autophagy in Atg7-deficient mice. J. Cell Biol 2005; 169: 425-434.

25. Kuma A, Hatano M, Matsui M, Yamamoto A, Nakaya H, Yoshimori T et al. The role of autophagy during the early neonatal starvation period. Nature 2004; 432: 1032-1036

26. Boya $\mathrm{P}$, Gonzalez-Polo RA, Casares N, Perfettini JL, Dessen P, Larochette N et al. Inhibition of macroautophagy triggers apoptosis. Mol Cell Biol 2005; 25: 1025-1040.

27. Lum JJ, Bauer DE, Kong M, Harris MH, Li C, Lindsten T et al. Growth factor regulation of autophagy and cell survival in the absence of apoptosis. Cell 2005; 120: 237-248.

28. Fung C, Lock R, Gao S, Salas E, Debnath J. Induction of autophagy during extracellular matrix detachment promotes cell survival. Mol Biol Cell 2008; 19: 797-806.

29. Karantza-Wadsworth V, Patel S, Kravchuk O, Chen G, Mathew R, Jin S et al. Autophagy mitigates metabolic stress and genome damage in mammary tumorigenesis. Genes Dev 2007; 21: 1621-1635.

30. Mathew R, Kongara S, Beaudoin B, Karp C, Bray K, Degenhardt K et al. Autophagy suppresses tumor progression by limiting chromosomal instability. Genes Dev 2007; 21 $1367-1381$. 
31. Paglin S, Hollister T, Delohery T, Hackett N, McMahill M, Sphicas E et al. A novel response of cancer cells to radiation involves autophagy and formation of acidic vesicles. Cancer Res 2001; 61: 439-444.

32. Ito H, Daido S, Kanzawa T, Kondo S, Kondo Y. Radiation-induced autophagy is associated with LC3 and its inhibition sensitizes malignant glioma cells. Int J Oncol 2005; 26 : $1401-1410$

33. Kim I, Rodriguez-Enriquez S, Lemasters J. Selective degradation of mitochondria by mitophagy. Arch Biochem Biophys 2007; 462: 245-253.

34. Carew J, Nawrocki S, Kahue C, Zhang H, Yang C, Chung L et al. Targeting autophagy augments the anticancer activity of the histone deacetylase inhibitor SAHA to overcome Bcr-Abl-mediated drug resistance. Blood 2007; 110: 313-322.

35. Nguyen T, Subramanian I, Kelekar A, Ramakrishnan S. Kringle 5 of human plasminogen, an angiogenesis inhibitor, induces both autophagy and apoptotic death in endothelial cells. Blood 2007; 109: 4793-4802

36. Qu X, Zou Z, Sun Q, Luby-Phelps K, Cheng P, Hogan R et al. Autophagy gene-dependent clearance of apoptotic cells during embryonic development. Cell 2007; 128: 931-946.

37. Inbal B, Bialik S, Sabanay I, Shani G, Kimchi A. DAP kinase and DRP-1 mediate membrane blebbing and the formation of autophagic vesicles during programmed cell death. J Cell Biol 2002; 157: 455-468.

38. Corradetti M, Guan K. Upstream of the mammalian target of rapamycin: do all roads pass through mTOR? Oncogene 2006; 25: 6347-6360.

39. Guertin D, Sabatini D. Defining the role of mTOR in cancer. Cancer Cell 2007; 12: 9-22.

40. Wouters B, Koritzinsky M. Hypoxia signaling through mTOR and the unfolded protein response in cancer. Nat Rev Cancer 2008; 8: 851-864.

41. Song G, Ouyang G, Bao S. The activation of Akt/PKB signaling pathway and cell survival. J Cell Mol Med 2005; 9: 59-71.

42. McCubrey J, Steelman L, Chappell W, Abrams S, Wong E, Chang F et al. Roles of the Raf/ MEK/ERK pathway in cell growth, malignant transformation and drug resistance. Biochim Biophys Acta 2007; 1773: 1263-1284.

43. Ballif $\mathrm{B}$, Blenis J. Molecular mechanisms mediating mammalian mitogen-activated protein kinase (MAPK) kinase (MEK)-MAPk survival signals. Cell Growth Diff 2001; 12 397-408.

44. Gozuacik D, Kimchi A. Autophagy as a cell death and tumor suppressor mechanism. Oncogene 2004; 23: 2891-2906.

45. Hanada T, Noda N, Satomi Y, Ichimura Y, Fujioka Y, Takao T et al. The Atg12-Atg5 conjugate has a novel E3-like activity for protein lipidation in autophagy. J Biol Chem 2007; 282: 37298-37302.

46. Fujita N, Itoh T, Omori H, Fukuda M, Noda T, Yoshimori T. The Atg16 complex specifies the site of LC3 lipidation for membrane biogenesis in autophagy. Mol Biol Cell 2008; 19 2092-2100.

47. Yousefi S, Perozzo R, Schmid I, Ziemiecki A, Schaffner T, Scapozza L et al. Calpainmediated cleavage of Atg5 switches autophagy to apoptosis. Nat Cell Biol 2006; 8 : 1124-1132.

48. Vaux DL, Cory S, Adams JM. Bcl-2 gene promotes haemopoietic cell survival and cooperates with c-myc to immortalize pre-B cells. Nature 1988; 335: 440-442.

49. Pattingre S, Tassa A, Qu X, Garuti R, Liang XH, Mizushima N et al. Bcl-2 antiapoptotic proteins inhibit Beclin 1-dependent autophagy. Cell 2005; 122: 927-939.

50. Levine B, Sinha S, Kroemer G. Bcl-2 family members: dual regulators of apoptosis and autophagy. Autophagy 2008; 4: 6.

51. Takahashi Y, Karbowski M, Yamaguchi $H$, Kazi A, Wu J, Sebti S et al. Loss of Bif-1 suppresses Bax/Bak conformational change and mitochondrial apoptosis. Mol Cell Biol 2005; 25: 9369-9382.

52. Noble $\mathrm{C}$, Dong J, Manser E, Song H. BCL-XL and UVRAG cause a monomer-dimer switch in beclin1. J Biol Chem 2008; 283: 26274-26282.

53. Zalckvar E, Berissi H, Mizrachy L, Idelchuk Y, Koren I, Eisenstein M et al. DAPk-mediated phosphorylation on the $\mathrm{BH} 3$ domain of Beclin-1 promotes dissociation of Beclin-1 from $\mathrm{Bc}$ $\mathrm{XL}$ to induce autophagy. EMBO Rep 2009; 10: 285-292.

54. Wei Y, Pattingre S, Sinha S, Bassik M, Levine B. JNK1-mediated phosphorylation of Bcl-2 regulates starvation-induced autophagy. Mol Cell 2008; 30: 678-688.

55. Zhang H, Bosch-Marce M, Shimoda LA, Tan YS, Baek JH, Wesley JB et al. Mitochondria autophagy is an HIF-1-dependent adaptive metabolic response to hypoxia. $\mathrm{J}$ Biol Chem 2008; 283: 10892-10903

56. Mellor $\mathrm{H}$, Harris A. The role of the hypoxia-inducible BH3-only proteins BNIP3 and BNIP3L in cancer. Cancer Metastasis Rev 2007; 26: 553-566.

57. Zhu J, Horbinski C, Guo F, Watkins S, Uchiyama Y, Chu C. Regulation of autophagy by extracellular signal-regulated protein kinases during 1-methyl-4-phenylpyridinium-induced cell death. Am J Pathol 2007; 170: 75-86.

58. Scarlatti F, Maffei R, Beau I, Codogno P, Ghidoni R. Role of non-canonical Beclin 1 independent autophagy in cell death induced by resveratrol in human breast cancer cells. Cell Death Diff 2008; 15: 1318-1329.

59. Scarlatti F, Maffei R, Beau I, Ghidoni R, Codogno P. Non-canonical autophagy. Autophagy 2008; 4: 1083-1085

60. Ruvolo PP, Deng X, Ito T, Carr BK, May WS. Ceramide induces Bcl2 dephosphorylation via a mechanism involving mitochondrial PP2A. J Biol Chem 1999; 274: 20296-20300.

61. Fridman JS, Lowe SW. Control of apoptosis by p53. Oncogene 2003; 22: 9030-9040.

62. Crighton D, Wilkinson S, O'Prey J, Syed N, Smith P, Harrison P et al. DRAM, p53-induced modulator of autophagy, is critical for apoptosis. Cell 2006; 126: 121-134.
63. Tasdemir E, Maiuri M, Galluzzi L, Vitale I, Djavaheri-Mergny M, D'Amelio M et al. Regulation of autophagy by cytoplasmic p53. Nat Cell Biol 2008; 10: 676-687.

64. Reef S, Zalckvar E, Shifman O, Bialik S, Sabanay H, Oren M et al. A short mitochondria form of p19ARF induces autophagy and caspase-independent cell death. Mol Cell 2006; 22: $463-475$.

65. Reef S, Shifman O, Oren M, Kimchi A. The autophagic inducer smARF interacts with and is stabilized by the mitochondrial p32 protein. Oncogene 2007; 26: 6677-6683.

66. Abida W, Gu W. p53-dependent and p53-independent activation of autophagy by ARF. Cancer Res 2008; 68: 352-357.

67. Reef S, Kimchi A. Nucleolar p19ARF, unlike mitochondrial smARF, is incapable of inducing p53 independent autophagy. Autophagy 2008; 4: 866-869.

68. Pimkina J, Humbey O, Zilfou J, Jarnik M, Murphy M. ARF induces autophagy by virtue of interaction with Bcl-xl. J Biol Chem 2009; 284: 2803-2810.

69. Bialik S, Kimchi A. The death-associated protein kinases: structure, function and beyond. Annu Rev Biochem 2006; 75: 189-210.

70. Wang WJ, Kuo JC, Yao CC, Chen RH. DAP-kinase induces apoptosis by suppressing integrin activity and disrupting matrix survival signals. J Cell Biol 2002; 159: 169-179.

71. Kuo J, Wang W, Yao C, Wu P, Chen R. The tumor suppressor DAPK inhibits cell motility by blocking the integrin-mediated polarity pathway. J Cell Biol 2006; 172: 619-631.

72. Deiss LP, Feinstein E, Berissi $\mathrm{H}$, Cohen $\mathrm{O}$, Kimchi A. Identification of a novel serine/ threonine kinase and a novel $15-\mathrm{kD}$ protein as potential mediators of the gamma interferoninduced cell death. Genes Dev 1995; 9: 15-30.

73. Kang C, You Y, Avery L. Dual roles of autophagy in the survival of Caenorhabditis elegans during starvation. Genes Dev 2007; 21: 2161-2171.

74. Bialik S, Bresnick AR, Kimchi A. DAP-kinase-mediated morphological changes are localization dependent and involve myosin-II phosphorylation. Cell Death Differ 2004; 11: 631-644.

75. Kuo JC, Lin JR, Staddon JM, Hosoya H, Chen RH. Uncoordinated regulation of stress fibers and focal adhesions by DAP kinase. J Cell Sci 2003; 116: 4777-4790.

76. Harrison B, Kraus M, Burch L, Stevens C, Craig A, Gordon-Weeks P et al. DAPK-1 binding to a linear peptide motif in MAP1B stimulates autophagy and membrane blebbing. $J$ Biol Chem 2008; 283: 9999-10014.

77. Halpain S, Dehmelt L. The MAP1 family of microtubule-associated proteins. Genome Biol 2006; 7: 224

78. Raveh T, Droguett G, Horwitz MS, DePinho RA, Kimchi A. DAP kinase activates a p19ARF/p53-mediated apoptotic checkpoint to suppress oncogenic transformation. Nat Cell Biol 2001; 3: 1-7.

79. Chen $\mathrm{CH}$, Wang WJ, Kuo JC, Tsai HC, Lin JR, Chang ZF et al. Bidirectional signals transduced by DAPK-ERK interaction promote the apoptotic effect of DAPK. EMBO J 2005; 24: 294-304.

80. Eisenberg-Lerner A, Kimchi A. DAP-kinase regulates JNK signaling by binding and activating protein kinase D under oxidative stress. Cell Death Differ 2007; 14: 1908-1915.

81. laquinta $\mathrm{P}$, Lees J. Life and death decisions by the E2F transcription factors. Curr Opin Cell Biol 2007; 19: 649-657.

82. Polager S, Ofir M, Ginsberg D. E2F1 regulates autophagy and the transcription of autophagy genes. Oncogene 2008; 27: 4860-4864.

83. Jin S. Autophagy, mitochondrial quality control, and oncogenesis. Autophagy 2006; 2 : $80-84$

84. Graeber T, Osmanian C, Jacks T, Housman D, Koch C, Lowe S et al. Hypoxia-mediated selection of cells with diminished apoptotic potential in solid tumours. Nature 1996; 379: 88-91.

85. Degenhardt K, Mathew R, Beaudoin B, Bray K, Anderson D, Chen G et al. Autophagy promotes tumor cell survival and restricts necrosis, inflammation, and tumorigenesis. Cancer Cell 2006; 10: 51-64.

86. Kim K, Hwang M, Moretti L, Jaboin J, Cha Y, Lu B. Autophagy upregulation by inhibitors of caspase- 3 and mTOR enhances radiotherapy in a mouse model of lung cancer. Autophagy 2008; 4: 659-668.

87. Shinohara E, Cao C, Niermann K, Mu Y, Zeng F, Hallahan D et al. Enhanced radiation damage of tumor vasculature by mTOR inhibitors. Oncogene 2005; 24: 5414-5422.

88. Bae D, Lu S, Taglienti C, Mercurio A. Metabolic stress induces the lysosomal degradation of neuropilin-1 but not neuropilin-2. J Biol Chem 2008; 283: 28074-28080.

89. Bialik S, Kimchi A. DAP-kinase as a target for drug design in cancer and diseases associated with accelerated cell death. Semin Cancer Biol 2004; 14: 283-294.

90. Liang C, Feng P, Ku B, Dotan I, Canaani D, Oh B et al. Autophagic and tumour suppressor activity of a novel Beclin1-binding protein UVRAG. Nat Cell Biol 2006; 8: 688-699.

91. Kim M, Jeong E, Ahn C, Kim S, Lee S, Yoo N. Frameshift mutation of UVRAG, an autophagy-related gene, in gastric carcinomas with microsatellite instability. Hum Pathol 2008; 39: 1059-1063.

92. Bekri S, Adelaide J, Merscher S, Grosgeorge J, Caroli-Bosc F, Perucca-Lostanlen D et al. Detailed map of a region commonly amplified at $11 q 13 \rightarrow q 14$ in human breast carcinoma. Cytogenet Cell Genet 1997; 79: 125-131.

93. Ionov Y, Nowak N, Perucho M, Markowitz S, Cowell J. Manipulation of nonsense mediated decay identifies gene mutations in colon cancer cells with microsatellite instability. Oncogene 2004; 23: 639-645.

94. Itakura E, Kishi C, Inoue K, Mizushima N. Beclin 1 forms two distinct phosphatidylinositol 3 kinase complexes with mammalian Atg14 and UVRAG. Mol Biol Cell 2008; 19: 5360-5372.

95. Liang C, Lee J, Inn K, Gack M, Li Q, Roberts E et al. Beclin1-binding UVRAG targets the class $C$ Vps complex to coordinate autophagosome maturation and endocytic trafficking. Nat Cell Biol 2008; 10: 776-787. 